Bull. Nov. Comp. Center, Comp. Science, 40 (2016), 1-14

(C) 2016 NCC Publisher

\title{
Algorithms and software for the analysis of disordering the structure of cellular walls
}

\author{
G.B. Abdikerimova, A.L. Bychkov, Wei Xin Yu, F.A. Murzin, \\ N.E. Russkikh, S.S. Khairulin, E.I. Ryabchikova
}

\begin{abstract}
The purpose of this work is the development and search of the analysis algorithms for textural features and various orthonormal spectral decompositions of the images obtained by the transmission electronic microscopy. The research is carried out for the Institute of Solid State Chemistry and Mechanochemistry of the Siberian Branch of the Russian Academy of Sciences. Its main focus is on working out software tools for the analysis of above-mentioned micro-photos. Suitable algorithms have been selected and the corresponding program tools have been created.
\end{abstract}

Keywords: image processing, textural features, orthogonal transformations, analysis of micro-photos, electronic microscopy, plant raw materials.

\section{Introduction}

The basis for conducting this research is the Contract between the Institute of Solid State Chemistry and Mechanochemistry, SB RAS, and A.P. Ershov Institute of Informatics Systems, SB RAS.

The work is carried out under the Agreement No. 16-13-10200 of May 12, 2016 between the Russian Science Foundation, project manager and organization that has received a grant for fundamental scientific research and exploratory research on the project "Controlled change in the structure and composition of plant raw materials by mechanochemical methods to intensify the extraction of biologically active compounds".

The work is devoted to the analysis of textures. The source is the microphotos of raw plant materials ground in special mills. The aim of the work is to determine the porosity, chemical reactivity, and other parameters of raw materials from micro-photos, which has not been accomplished to the full because it requires deeper research. We have focused mainly on the development of software tools for image analysis, namely, programs for the analysis of textures with the help of sets of texture attributes (19 features) and spectral transformations based on orthogonal matrices (6 transforms). Also, we have carried out preliminary experiments on the application of $\mathrm{R} / \mathrm{S}$ analysis and fractal analysis. In the future, machine learning algorithms based, for example, on the neurocomputing approach, can be used to determine chem- 
ical reactivity. Having trained the system on a data set, we will be able to predict this parameter.

\section{Methods for the analysis of textural features}

Despite the ubiquity of textures in images, so far there is no unique formal approach to describing the texture and its strict definition. As a rule, methods for texture analysis are developed for each individual case.

In [1], a texture is understood as a "spatial organization of elements within part of the surface". It also explains that this organization is due to a certain statistical distribution of the intensity of gray tones or tones of different colors. A site can be considered as a texture if the number of intensity changes or color changes observed on it is sufficiently large. In [2], the texture is called "a surface area organized in some way". In [3], the texture is defined as a matrix or a fragment of the spatial properties of sections of images with homogeneous statistical characteristics.

Textures can be divided into several classes as follows:

1. by origin: artificial (for example, graphic patterns) and natural (for example, grass, forest, land);

2. by the surface structure: structural, consisting of geometrically correct repeating elements, and stochastic, formed by a sequence of random elements;

3. by the relative dimensions of texture elements: fine-grained and coarsegrained;

4. by the shape of texture elements: wavy, spotted, irregular, ruled, and so on $[1]$.

It follows from the above definitions and characteristics that a texture is a certain area of an image that has homogeneous statistical characteristics. This means that each texture of a given class can be described using a characteristic property common to all textures of the given class [1]. Such properties are called texture attributes. Textural features play an important role when an image is divided into separate areas. Let us consider in more detail the system of features.

In this problem, gray (halftone) images are considered. Thus, the image is given in the form of a matrix whose values are the brightness values of pixels in the range from 0 to 255 .

The standard approach to calculating the texture attributes is as follows. It is necessary to choose the so-called sliding window with the odd side: 3, 5, 7 pixels. The attribute is calculated inside the sliding window. The size of the local fragment is the carrier of texture properties. The value of the characteristic is written to a new matrix of the same size as the original one. 
In the new matrix, the value is written on the point with the coordinates equal to the coordinates of the center of the sliding window. The elements of the new matrix are obtained in a certain interval [A, B]. Further, this interval is linearly mapped into a segment $[0,255]$. After this, it is possible to visualize the result of calculating the texture feature.

Experiments have shown that the standard approach is not very informative in this case. Therefore, it was decided to use a non-standard approach. Namely, the texture windows are computed from large windows (including non-square ones) that the user can specify by selecting an area that may be of interest to him/her. That is, we are talking about the calculation of numerical characteristics relating to vast areas, including various kinds of artifacts. Nineteen most important textural features have been considered, and all of them have been implemented in the program.

\section{Main textural features}

\subsection{Features based on statistical characteristics}

As such features, we can use statistical moments of spatial distributions calculated as homogeneity measures from a one-dimensional histogram of signal values (first-order characteristics) and two-dimensional histograms of signal values (second-order characteristics). Thus, as the numerical estimates of the texture with respect to a one-dimensional histogram, the following statistical characteristics can be used [4]:

$\mathrm{k}$-th initial moment

$T_{1}^{k}=n^{-2} \sum_{i=1}^{1} \sum_{j=1}^{1}[f(i, j)]^{k}$

entropy

$T_{2}=-\sum_{g=0}^{N-1} F(g) \log _{10} F(g)$

energy

$T_{3}=\sum_{k=0}^{N-1}[F(g)]^{2}$

variation

$T_{4}=-\sum_{g=0}^{N-1}(g-\mu)^{2} F(g)$,

where $n$ is the size of the sliding window $(n=2 W+1)$ in pixels;

$f(i, j)$ is the brightness of the pixel at the point $(i, j)$ of the sliding window; $N$ is the number of gradations in the brightness of the image; 
$F(g)$ is the number of pixels with brightness $g$; and $\mu$ is average in the window $T_{m o m}^{1}$

The analysis shows that the texture estimates given above and calculated with respect to a one-dimensional frequency histogram do not take into account the relative positions of adjacent pixels in the sliding window and allow us to estimate only the group properties of the pixels that make up a particular landscape object in the aerospace image. Thus, these estimates are effective only for describing textures with not clearly expressed spatial regularity.

\subsection{Features taking into account the relative position}

To define the textural features that take into account mutual arrangement of pixels within the sliding window, an approach based on the use of an adjacency matrix (also called the gradient distribution matrix [4]) was applied. In what follows, we shall use the name of the adjacency matrix.

Let the analyzed image be a rectangle and have elements horizontally $N_{x}$ and vertically $N_{y}$. Also, $G=\{1,2, \ldots, N\}$ is a set of $N$ quantized brightness values from a set. Then the image is described by a function of the brightness values from the set $\mathrm{G}$, that is $f: L_{x} \times L_{y} \rightarrow G$, where $L_{x}=\left\{1,2, \ldots, N_{x}\right\}$ and $L_{y}=\left\{1,2, \ldots, N_{y}\right\}$ are the horizontal and vertical spatial regions, respectively. The adjacency matrix contains the relative $P_{i j}$ frequencies of the presence of adjacent elements located at a distance $d$ from each other in the image with luminances $i, j \in G$. Usually, horizontal $\left(\alpha=0^{\circ}\right)$, vertical $\left(\alpha=90^{\circ}\right)$ and cross-diagonal $\left(\alpha=45^{\circ}\right)$ and $\left(\alpha=135^{\circ}\right)$ pairs of elements are distinguished. It should be noted that these matrices are symmetric, i.e., $P(i, j, d, \alpha)=P(j, i, d, \alpha)$

On the basis of calculated adjacency matrices, it is possible to calculate directly the numerical estimates of a number of textural features [4]:

average

$T_{5}=\mu_{i}=\mu_{j}=\sum_{i=0}^{N-1}\left[i \sum_{j=0}^{N-1} P(i, j)\right]$;

energy

$T_{6}=\sum_{i=0}^{N-1} \sum_{j=0}^{N-1}[P(i, j)]^{2} ;$

variation

$T_{7}=\sigma_{i}^{2}=\sum_{i=0}^{N-1}\left[\left(i-\mu_{2}\right)^{2} \sum_{j=0}^{N-1} P(i, j)\right]$; and

homogeneity 
$T_{8}=\sum_{i=0}^{N-1} \sum_{j=0}^{N-1} P(i, j) /(1+|i-j|)$,

where $P(i, j)$ is the frequency of the appearance of two pixels in a sliding window with the brightness $i$ and $j$ at an angle $\alpha$ at a distance $d$.

$\sigma_{i}$ is a square deviation of brightness in a sliding window.

Statistical moments allow the formation of textural features that take into account the relative position of neighboring pixels in a sliding window and are accordingly effective for describing the textures with pronounced spatial regularity.

The second angular momentum

$T_{9}=\sum_{i=1}^{N} \sum_{i=1}^{N}(P(i, j) / M)^{2}$,

where $\mathrm{M}$ is the total number of pairs of adjacent elements (for example, for $\left.d=1, \alpha=0, M=2 N_{y}\left(N_{x}-1\right)\right)$ is a measure of homogeneity of the image and it takes in this case a minimum value.

The contrast, $T_{10}=\sum_{n=0}^{N-1} n^{2}\left[\sum_{i=1}^{N} \sum_{i=1}^{N} P(i, j) / M\right],|i-j|=n$, is defined by the magnitude of the local variations in the brightness of the image. As the number of local variations increases, the contrast increases.

The coefficient of correlation $T_{11}=\frac{\sum_{i=1}^{N} \sum_{j=1}^{N}\left[i j(P(i, j) / M)-m_{x} m_{y}\right]}{\sigma_{x}^{-1} \sigma_{y}^{-1}}$, where $m_{x}, m_{y}, \sigma_{x}, \sigma_{y}$ mean the values and root-mean-square deviations for $p_{x}(i)=\sum_{j=1}^{N} P(i, j) / M$ and $p_{y}(j)=\sum_{j=1}^{N} P(i, j) / M$, respectively. $T_{11}$ serves as a measure of the linearity of the regression dependence of brightness on the image.

The dispersion (variance) $T_{12}=\sum_{i=1}^{N} \sum_{j=1}^{N}(i-m)^{2}(P(i, j) / M)$ determines the brightness variations relative to the mean value.

The moment of the inverse difference

$$
T_{13}=\sum_{i=1}^{N} \sum_{j=1}^{N}\left[1+(i-j)^{2}\right]^{-1}(P(i, j) / M)
$$

is closely related to the contrast and reflects the degree of scatter of the elements of the gradient matrix around the main diagonal. This feature is an alternative to the contrast in the case of the influence of edge structures, 
since relatively large differences in brightness values contribute minimally to the final result.

The total average

$T_{14}=\sum_{n=2}^{2 N} n p_{+}(n)$,

where $p_{+}(n)=\sum_{i=1}^{N} \sum_{j=1}^{N} P(i, j) / M$ for $i+j=n, n=2,3, \ldots, 2 N$ is the histogram of the sums of brightness values. $T_{14}$ is determined by a histogram of sums of brightness $p_{+}(n)$ values over pairs of image elements, which is directly related to the adjacency matrix.

The total variance serves $T_{15}=\sum_{n=2}^{2 N}\left(n-T_{14}\right)^{2} p_{+}(n)$ as a measure of the brightness variation relative to the total average.

The total entropy for the histogram of sums of the brightness $T_{16}=\sum_{n=2}^{2 N} p_{+}(n) \log p_{+}(n)$

values is determined by the classical measure of statistical information theory and expresses the uneven distribution of the brightness properties of image elements.

The entropy $\left.T_{17}=-\sum_{i=1}^{N} \sum_{j=1}^{N} P(i, j) / M\right) \log P(i, j) / M$ is defined in the same way as the total entropy, but only for the adjacency matrix.

The differential dispersion $T_{18}=\sum_{i=1}^{N-1}\left[n-\sum_{m=0}^{N-1} p_{-}(m)\right]^{2} p_{-}(n)$ is expressed by means of a histogram of differences in the brightness

$p_{-}(n)=-\sum_{i=1}^{N} \sum_{j=1}^{N} P(i, j) / M$

values over pairs of image elements.

The differential entropy $T_{19}=-\sum_{n=0}^{N-1} p_{-}(n) \log p_{-}(n)$ is calculated as the total entropy and the entropy for the adjacency matrix, but for the histogram of differences in brightness values.

\section{Orthogonal transformations}

The spectral analysis is a powerful tool for analyzing signals and images, as it has long been noted that the spectrum is very sensitive to various changes in the structure of signals and images. 
To perform the spectral analysis, it is necessary to decompose a signal or image over frequencies. For this, different sets of basic functions are used. The corresponding algorithms are called transformations: cosine, Hadamard, Haar, slant, etc. We note that the Haar and Daubechies transforms are the simplest wavelet transforms. These methods, according to the signal processing theory, can be applied to stationary random processes, but we do not always have them. However, it is possible to choose the areas to be analyzed that can be considered conditionally stationary (in other words, quasi-stationary) and whose size is sufficient to obtain statistically reasonable results.

Another feature found in experiments with such algorithms is that the estimate is qualitative rather than quantitative, which also represents a certain value. Often there are no regulatory tables for the main parameters of signals or images, as is the case of cardiography, for example.

The use of decomposition algorithms for various basic functions can be considered a transition from one form of information display to another, more convenient, compact and informative.

In the one-dimensional case, the spectral transformation can be written in the form $H \vec{u}=\vec{\alpha}$, where $H$ is the transformation matrix whose rows form an orthonormal basis in the corresponding linear space; $\vec{u}$ is a vector, which is the sampling of the original signal; $\vec{\alpha}$ is a vector of spectral coefficients that characterize how a certain basic function (harmonic) is represented in the vector $\vec{u}$ (i.e., in the original signal).

In the two-dimensional case, i.e., for images, the spectral transformation is written in the form $H U H^{T}=A$, where $H^{T}$ is the transposed matrix, $U$ is the square fragment of the original image, is the matrix containing the spectral coefficients. That is, we suppose that the transformation is applied to a fragment of the image.

Usually various remarkable bases, i.e., matrices, are used. They allow you to "uncover" accurately the nature of signals and images, i.e., to understand their structure. Below is a list of the most interesting transformations.

\subsection{Cosine transform}

The discrete two-dimensional (matrix) cosine transform DCT is usually given by the formula [10]:

$$
\begin{gathered}
G_{i j}=\frac{1}{\sqrt{2 n}} C_{i} C_{j} \sum_{x=0}^{n-1} \sum_{y=0}^{n-1} p_{x y} \cos \left(\frac{(2 y+1) j \pi}{2 n}\right) \cos \left(\frac{(2 x+1) i \pi}{2 n}\right), \\
C_{f}=\left\{\begin{array}{l}
1 / \sqrt{2}, f=0 \\
0, f>0 .
\end{array}\right.
\end{gathered}
$$

Here $p_{x y}$ is the brightness of the pixel with the coordinates $x, y$. That 
is, this formula immediately represents a record of the multiplication result for the matrices $H U H^{T}$.

\subsection{The Hadamard transform}

The papers $[11,12]$ describe the methods for constructing normalized Hadamard matrices known as Paley constructions.

Definition 1. Let $p$ be a prime number, $p \neq 2, \alpha$, and $n$ be an arbitrary integer not divisible by $p$. The Legendre symbol $(\alpha / p)$ is put to be equal to 1 if the equation $x^{2} \equiv \alpha(\bmod p)$ has a solution; and 1 , otherwise [11].

As is known [13], the following formula is valid:

$$
(\alpha / p)=-1^{M}, M=\sum_{x=1}^{p_{i}}\left[\frac{2 \alpha x}{p}\right], p_{1}=\frac{1}{2}(p-1) .
$$

Here the square brackets denote the integer part of division.

Definition 2. If $A=\left(a_{i j}\right)$ is an $(n \times n)$ - matrix and $B=\left(b_{k s}\right)$ is an $(m \times m)$ - matrix, then the Kronecker product $(A \times B)$ is called an $(n m \times n m)$ matrix

$$
A \times B=\left(\begin{array}{cccc}
a_{00} B & a_{01} B & \cdots & a_{0 n-1} B \\
a_{10} B & a_{11} B & \cdots & a_{1 n-} B \\
\vdots & \vdots & \ddots & \vdots \\
a_{n-1,0} B & a_{n-1,1} B & \cdots & a_{n-1, n-1} B
\end{array}\right)
$$

Further, we give a brief description of the two classes of matrices:

1) The matrices of the order $n=2^{k}$ are determined by induction.

For $k=1$ we set

$H_{1}=\left(\begin{array}{cc}1 & 1 \\ 1 & -1\end{array}\right)$.

If $H_{k}$ it is already defined, then let

$H_{k+1}=H_{1} \times H_{k}$

2) Matrices of the order $n=p+1$, where $p \equiv 3(\bmod 4)$ is a prime number.

We set $\chi(k)=(k / p)$ and

$\alpha_{i j}=+1,(i=0$ or $j=0)$

$\alpha_{i j}=\chi(j-i),(1 \leq i, j \leq p, i \neq j)$

$\alpha_{i i}=-1,(1 \leq i \leq p)$

Proposition. The following relations hold:

a) $\alpha_{i j}=-\alpha_{i j},(i, j \geq 1, i \neq j)$,

b) $\alpha_{i j}=\alpha_{i+k, j+k},(i, j \geq 1, i \neq j)$,

c) $\alpha_{i, i+k}=\chi(k),(i, k \geq 1)$, 
d) $\alpha_{i, i+k}=-\alpha_{i, i+p-k},(i, k \geq 1)$.

The proof follows directly from the well-known properties [13] of the Legendre symbol.

The first equality in this sentence means that the matrix $A$ is antisymmetric. The second shows that, on any line parallel to the main diagonal, all elements are equal. The third equality gives, in particular, the structure of the first line: $\langle 1,-1, \chi(1), \chi(2), \ldots\rangle$. From the fourth equality, it follows that the segment of the first line $\left\langle\alpha_{12}, \alpha_{13}, \ldots, \alpha_{1, p-1}\right\rangle$, lying above the main diagonal, has the antisymmetry property. Thus, the whole segment is restored along its half.

\subsection{The Haar transformation}

The Haar transformation is based on the orthogonal Haar matrix [13]. Below is an example of the eighth order orthonormal Haar matrix

$$
H_{8}=\frac{1}{\sqrt{8}}\left(\begin{array}{cccccccc}
1 & 1 & 1 & 1 & 1 & 1 & 1 & 1 \\
1 & 1 & 1 & 1 & -1 & -1 & -1 & -1 \\
\sqrt{2} & \sqrt{2} & -\sqrt{2} & -\sqrt{2} & 0 & 0 & 0 & 0 \\
0 & 0 & 0 & 0 & \sqrt{2} & \sqrt{2} & -\sqrt{2} & -\sqrt{2} \\
2 & -2 & 0 & 0 & 0 & 0 & 0 & 0 \\
0 & 0 & 2 & -2 & 0 & 0 & 0 & 0 \\
0 & 0 & 0 & 0 & 2 & -2 & 0 & 0 \\
0 & 0 & 0 & 0 & 0 & 0 & 2 & -2
\end{array}\right)
$$

Higher-order Haar matrices are constructed according to the same rules as matrices $H_{4}$ and $H_{8}$. The Haar transformation can be considered as a process of discretization of the original signal, in which the step of digitization is halved with the transition to the next block of lines.

\subsection{Slant transformation}

$$
S_{N}=\frac{1}{\sqrt{2}}\left(\begin{array}{cccccc}
1 & 0 & & 1 & 0 & \\
a_{N} & b_{N} & & -a_{N} & b_{N} & \\
0 & E_{N / 2-2} & 0 & & E_{N / 2-2} \\
0 & 1 & & 0 & -1 & 0 \\
-b_{N} & a_{N} & & b_{N} & a_{N} & \\
0 & E_{N / 2-2} & 0 & & E_{N / 2-2}
\end{array}\right)\left(\begin{array}{cc}
S_{N / 2} & 0 \\
0 & S_{N / 2}
\end{array}\right)
$$

where $E_{K}$ is the unit matrix of the $K$-th order.

The constants $a_{N}$ and $b_{N}$ can be found from the recurrence relations 


$$
\begin{gathered}
a_{2}=1 \\
b_{N}=\left(1+4\left(a_{N / 2}\right)^{2}\right)^{-\frac{1}{2}} \\
a_{N}=2 b_{N} a_{N} / 2
\end{gathered}
$$

or by the formulas

$$
\begin{aligned}
& a_{2 N}=\left(\frac{3 N^{2}}{4 N^{2}-1}\right)^{\frac{1}{2}} \\
& b_{2 N}=\left(\frac{N^{2}-1}{4 N^{2}-1}\right)^{\frac{1}{2}} .
\end{aligned}
$$

\subsection{Transformation of Daubechies-4}

The Daubechies-4 transformation is given (see $[13,14]$ ) by means of the following matrix

$$
M=\sqrt{2}\left[\begin{array}{ccccccccc}
h_{0} & h_{1} & h_{2} & h_{3} & & & & \\
& & h_{0} & h_{1} & h_{2} & h_{3} & & \\
& & & & h_{0} & h_{1} & h_{2} & h_{3} \\
h_{2} & h_{3} & & & & & h_{0} & h_{1} \\
h_{3} & -h_{2} & h_{1} & -h_{0} & & & & \\
& & h_{3} & -h_{2} & h_{1} & -h_{0} & & \\
& & & & h_{3} & -h_{2} & h_{1} & -h_{0} \\
h_{1} & -h_{0} & & & & & h_{3} & -h_{2}
\end{array}\right] .
$$

The elements of the matrix are calculated by the formulas given below:

$$
h_{0}=(1+\sqrt{3}) / 8, h_{1}=(3+\sqrt{3}) / 8, h_{2}=(3-\sqrt{3}) / 8, h_{3}=(1-\sqrt{3}) / 8 .
$$

\section{Program for calculation of textural features}

The goal of the program is to carry out computer experiments and accumulate quantitative data (for example, textural attribute values) for different sections of the images of cell walls treated at different temperatures. At the input, the program receives the image in any of the standard formats (bmp, jpeg, tiff, png), and a dialog box opens allowing you to select rectangular areas of an arbitrary size of interest. The coordinates and sizes of the selected areas of interest and the values of texture attributes are written to the csv file. The developed program allows you to calculate 19 features (described in Section 2) in the selected window. The program has two modes of processing the image. The first mode allows marking an image highlighting a few areas of interest; each area is limited to a fixed rectangle whose border is indicated by a unique color. In this case, the pop-up windows display the values of the texture attributes for the selected area of the image. The second mode allows working with one limiting contour and changing the 
size of the contour dynamically or moving it to follow the changes in the characteristic values. The image areas are selected with a computer mouse when the left mouse button is pressed. Figure 1 shows how the program works.

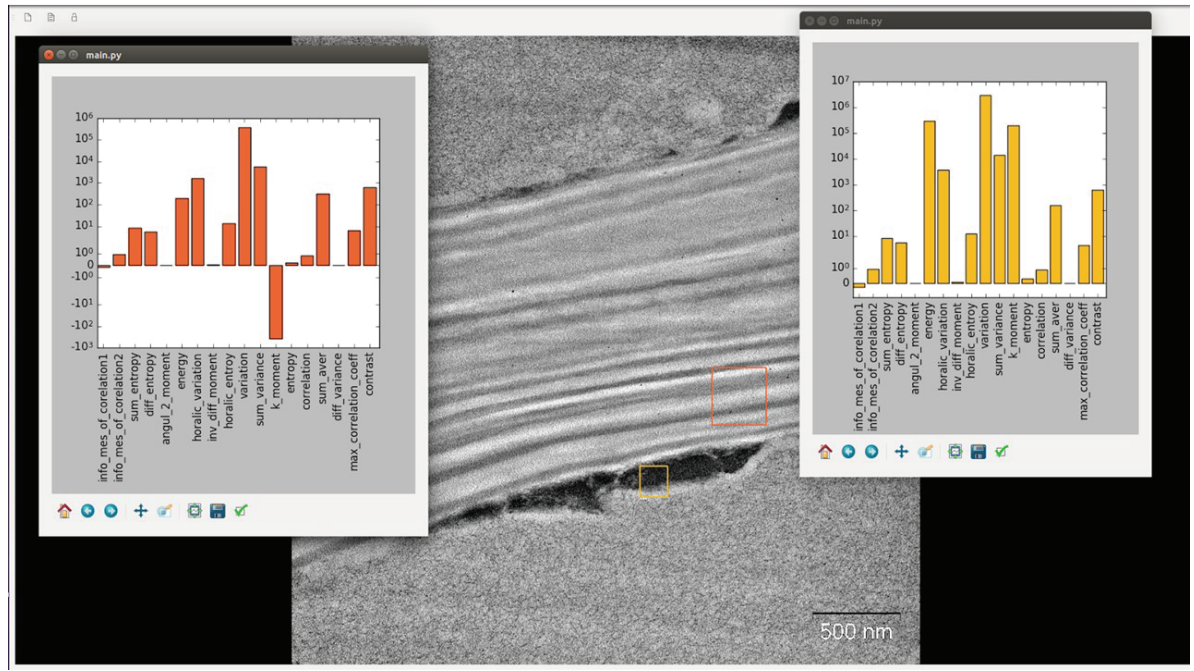

Figure 1. Selection of multiple image areas. The diagrams show the values of the corresponding textural features

\section{Program for calculation of orthogonal transformations}

The program is implemented in the Matlab environment and allows us to calculate spectral transformations of six types: 1) cosine, 2) Hadamard of the order $2^{n}$, 3) Hadamard of the order $n=p+1, p \equiv 3(\bmod 4)$ is a prime number, i.e., based on the Legendre symbol, 4) Haar, 5) slant, and 6) Daubechies 4.

Before starting the main program with the help of an auxiliary program, a working window is selected (Figure 2). Namely, a small window appears on the image under study. It can be moved where necessary with the help of a mouse, but its size cannot be changed, that is, we cannot compress or expand it. At the top, when the window is moving, four numbers are displayed in rectangular brackets. These are the coordinates of the bottom left corner and the size of the window. Then you can take the first two numbers (the $x$ and $y$ coordinates) and manually enter them into the second program that will perform the calculation. The main program is called main.m.

All the code of the main program can be manually inserted into the working environment of Matlab, and immediately the results - graphs and seven text files will appear. The graphs of the results of the transformations 


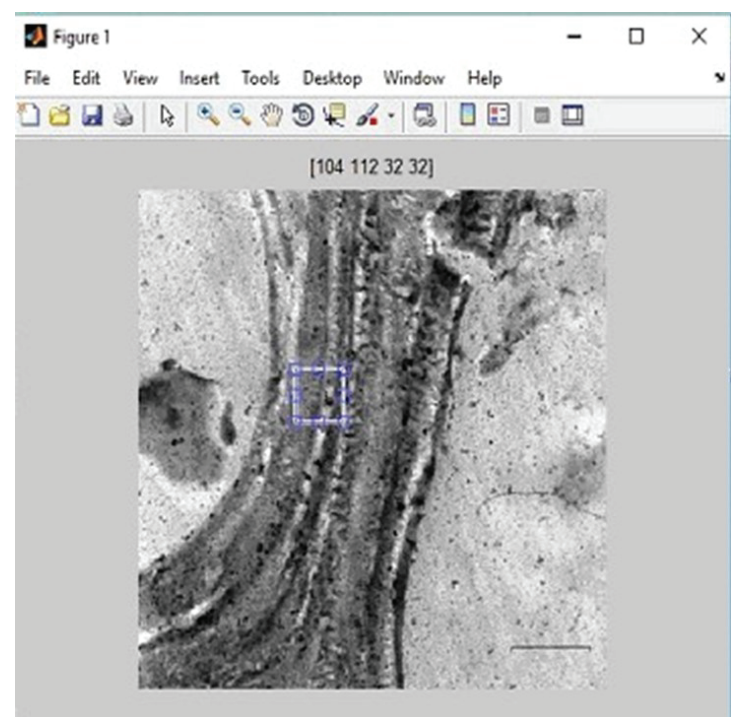

Figure 2. A screen of the auxiliary program

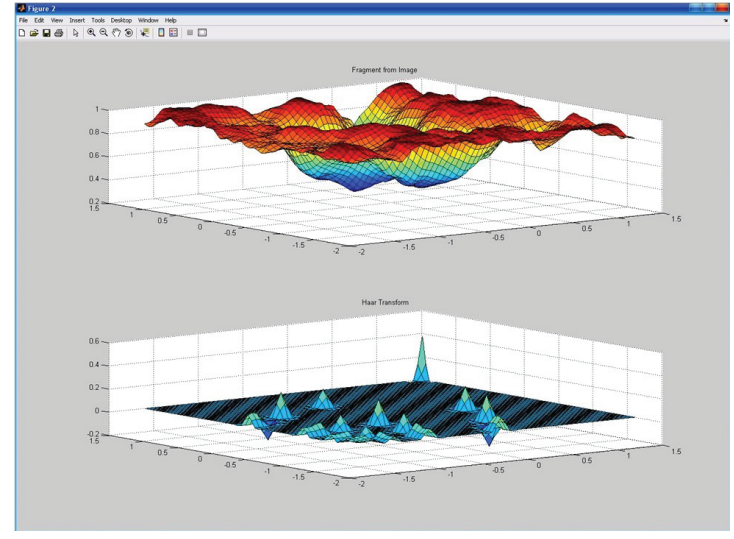

Figure 3. The graph of the original brightness function in the window and the result of the Haar transformation

will be presented each in a separate window (Figure 3) and all simultaneously in one window. Matlab allows the graphics to be rotated and viewed from different angles. In the text files, the original data and the results of the above six transformations are automatically saved.

\section{Conclusion}

The work was carried out for the Institute of Solid State Chemistry and Mechanochemistry of the SB RAS. The work is devoted to the study of 
texture images. The source data are microphotos of plant raw materials ground in special mills. The ultimate goal of the work is to determine their porosity, chemical reactivity and other parameters. This paper does not solve this task completely because it requires more in-depth studies. Here, we are talking about the computation of various kinds of textural features and other characteristics of images. In the future, the feature vectors can be "linked" to chemical reactivity and a software system can be trained using various machine-learning algorithms, such as the neurocomputing approach. A large set of methods for studying textures have been analyzed, and the most informative ones have been selected.

The main goal of our work is the creation of software tools and the result is presented below.

1. A program for calculating various textural features. The program is intended to conduct computer experiments and accumulate quantitative data (the values of textural features) for different sections of images of cell walls treated at different temperatures. The program has feature-rich graphical user interfaces and allows us to select rectangular areas of interest of an arbitrary size for which the values of texture attributes are displayed in the form of diagrams in separate pop-up windows. A total of 19 texture attributes can be calculated and the results of calculations can be saved.

2. A program implemented in the Matlab environment that allows spectral transformations of six types: 1) cosine, 2) Hadamard of the order $2^{n}$, 3 ) Hadamard of the order $n=p+1$, where $p \equiv 3(\bmod 4)$ is a prime number, i.e., based on the Legendre symbol, 4) Haar, 5) slant, and 6) Daubechies-4. The mathematical part is implemented, but the program has no GUI yet. Later, it will be built into the first program, and corresponding interfaces will appear.

3. The prototypes of programs intended to perform R / S analysis and fractal analysis [7-9]. The initial experiments have shown interesting results that can be used in practice, namely, to support research in chemistry. Note that similar statements are found in the scientific literature devoted to the study of lignin $[5,6]$ but these results are not reflected in this paper.

\section{References}

[1] Andreev G.A, Bazarsky O.V., Glauberman A.S., Kolesnikov A.I., Korzhik Yu.V., Khlyavich Ya.L. Analysis and synthesis of random spatial textures // Foreign Radioelectronics. - 1984. - No. 2. - P. 3-33 (In Russian).

[2] Haralik R.M. Statistical and structural approaches to the description of textures // Proc. IEEE. - 1979. - Vol. 67, No. 5. - P. 98-119.

[3] Potapov A.A. New information technologies based on probabilistic textural and fractal signs in radar detection of low contrasting targets // Radio Engineering and Electronics. - 2003. - Vol. 48, No. 9. - P. 1101-1119 (In Russian). 
[4] Kolodnikova N.V. Survey of texture attributes for image recognition problems // Reports of TUSUR. Automated Systems for Information Processing, Control and Design. - 2004. - P. 117-118 (In Russian).

[5] Karmanov A.P. Lignin. Structural organization and self-organization // Chemistry of Plant Raw Material. - 1999. - No 1. - P. 65-74 (In Russian).

[6] Karmanov A.P., Matveev D.V. Simulation of Lignin Biosynthesis // Problems of Wood Chemistry and Timber Chemistry. - Institute of Chemistry of the Komi Scientific Center of the Ural Branch of the Russian Academy of Sciences. Syktyvkar, 1997. P. 5862 (In Russian).

[7] Peters E. Chaos and Order in the Capital Markets. - M.: Mir, 2000 (In Russian).

[8] Barantsev R.G. Synergetics in Modern Natural Science. - M .: Editorial URSS, 2003 (In Russian).

[9] Petrov V.V. The Structure of Teletraffic and the Algorithm for Ensuring the Quality of Service under the Influence of the Self-Similarity Effect. - Thes... cand. engineering. - M: Izd. of the Moscow Power Engineering Institute, 2004 (In Russian).

[10] Salomon D. Compression of Data, Images and Sound. - M.: Technosphere, 2004 (In Russian).

[11] Hall M. Combinatorics. - M.: Mir, 1970 (In Russian).

[12] Paley R.E.A.C. On orthogonal matrices // J. Math. and Phys.- 1933. - No. 12. - P. 321-333.

[13] Vorobiev V.I., Gribunin V.G. Theory and Practice of Wavelet Transform. St. Petersburg: Publishing House of the Military Communication University, 1999 (In Russian).

[14] Daubechies I. Ten Lectures on Wavelets. - M.: Regular and chaotic dynamics, 2001 (In Russian). 